\title{
Baicalein, a Component of Scutellaria baicalensis, Attenuates Kidney Injury Induced by Myocardial Ischemia and Reperfusion
}

Authors

Affiliations
Chang-Chi Lai ${ }^{1,2,3}$, Po-Hsung Huang ${ }^{3,4,5}$, An-Han Yang ${ }^{6,7}$, Shu-Chiung Chiang ${ }^{8}$, Chia-Yu Tang ${ }^{1,2,3}$, Kuo-Wei Tseng ${ }^{9}$, Cheng-Hsiung Huang ${ }^{1,2}$

The affiliations are listed at the end of the article

\author{
Key words \\ - Scutellaria baicalensis \\ - Lamiaceae \\ - acute kidney injury \\ - apoptosis \\ - baicalein \\ - myocardial ischemia \\ and reperfusion \\ - prosurvival kinases
}

$\begin{array}{ll}\text { received } & \text { April 20, 2015 } \\ \text { revised } & \text { June 9, 2015 } \\ \text { accepted } & \text { Sept. 7, 2015 }\end{array}$

Bibliography

DOI http://dx.doi.org/

10.1055/s-0035-1558114

Published online November 9,

2015

Planta Med 2016; 82: 181-189

(C) Georg Thieme Verlag KG

Stuttgart · New York .

ISSN 0032-0943

\section{Correspondence}

Cheng-Hsiung Huang, MD, PhD

Division of Cardiovascular

Surgery

Taipei Veterans General Hospital

\#201, Section 2, Shih-Pai Road

Taipei, 11217

Taiwan

Phone: + 886228757495

Fax: + 886228757534

chhuang@vghtpe.gov.tw

\section{Abstract \\ $\nabla$}

Acute kidney injury is a common and severe complication of acute myocardial infarction and cardiac surgery. It results in increased mortality, morbidity, and duration of hospitalization. Baicalein is a component of the root of Scutellaria baicalensis, which has traditionally been used to treat cardiovascular and liver diseases in Asia. In this study, we investigated whether baicalein can attenuate kidney injury induced by myocardial ischemia and reperfusion in rats. Myocardial ischemia and reperfusion, induced by a 40 -minute occlusion and a 3-hour reperfusion of the left anterior descending coronary artery, significantly increased blood urea nitrogen and creatinine levels in addition to causing histological changes in the kidneys. Kidney apoptosis was also significantly increased. Furthermore, myocardial ischemia and reperfusion significantly increased the serum levels of tumor necrosis factor- $\alpha$, interleukin- 1 , and interleukin- 6 as well as the tumor necrosis factor- $\alpha$ levels in the kidneys. Intravenous pretreatment with baicalein (in doses of 3,10 , or $30 \mathrm{mg} /$ $\mathrm{kg}$ ), however, significantly reduced the increases in the creatinine level, renal histological damage, and apoptosis induced by myocardial ischemia and reperfusion. In addition, the increases in the serum levels of tumor necrosis factor- $\alpha$, interleukin-1, and interleukin- 6 , and of tumor necrosis factor- $\alpha$ in the kidneys were significantly reduced. Western blot analysis revealed that baica- lein significantly increased Bcl-2 and reduced Bax in the kidneys. The phosphorylation of Akt and extracellular signal-regulated kinases 1 and 2 was also significantly increased. In conclusion, baicalein significantly attenuates kidney injury induced by myocardial ischemia and reperfusion. The underlying mechanisms might be related to the inhibition of apoptosis, possibly through the reduction of tumor necrosis factor- $\alpha$ production, the modulation of Bcl-2 and Bax, and the activation of Akt and extracellular signal-regulated kinases 1 and 2 .

\section{Abbreviations \\ $\checkmark$ \\ AAR: $\quad$ area at risk \\ AKI: acute kidney injury \\ BUN: blood urea nitrogen \\ CAO: coronary artery occlusion \\ CAR: coronary artery reperfusion \\ ELISA: enzyme-linked immunosorbent assay \\ ERK1/2: extracellular signal-regulated kinases 1 and 2 \\ IL-1: interleukin-1 \\ IL-6: interleukin-6 \\ I/R: $\quad$ ischemia and reperfusion \\ MI: myocardial infarct/infarction \\ TNF- $\alpha$ : $\quad$ tumor necrosis factor- $\alpha$}

TUNEL: terminal deoxynucleotidyl transferase-mediated dUTP nick-end labeling

\section{Introduction}

\section{$\nabla$}

AKI is a common and severe complication of cardiac surgery and acute MI, and is associated with increased morbidity, mortality, and medical costs $[1,2]$. Myocardial I/R occurs in cardiac surgery and revascularization treatments for acute MI. We recently reported that myocardial $\mathrm{I} / \mathrm{R}$ induces AKI in rats [3]. Myocardial $\mathrm{I} / \mathrm{R}$ elicits the production of proinflammatory cytokines, such as TNF- $\alpha$, IL-1, and IL-6, resulting in necrosis and apoptosis [35]. Apoptosis, in turn, has been shown to contribute to the development of AKI [6].

Baicalein ( $\bullet$ Fig. 1 A) is a component of the root of Scutellaria baicalensis Georgi (Lamiaceae), which has traditionally been used to treat cardiovascular 


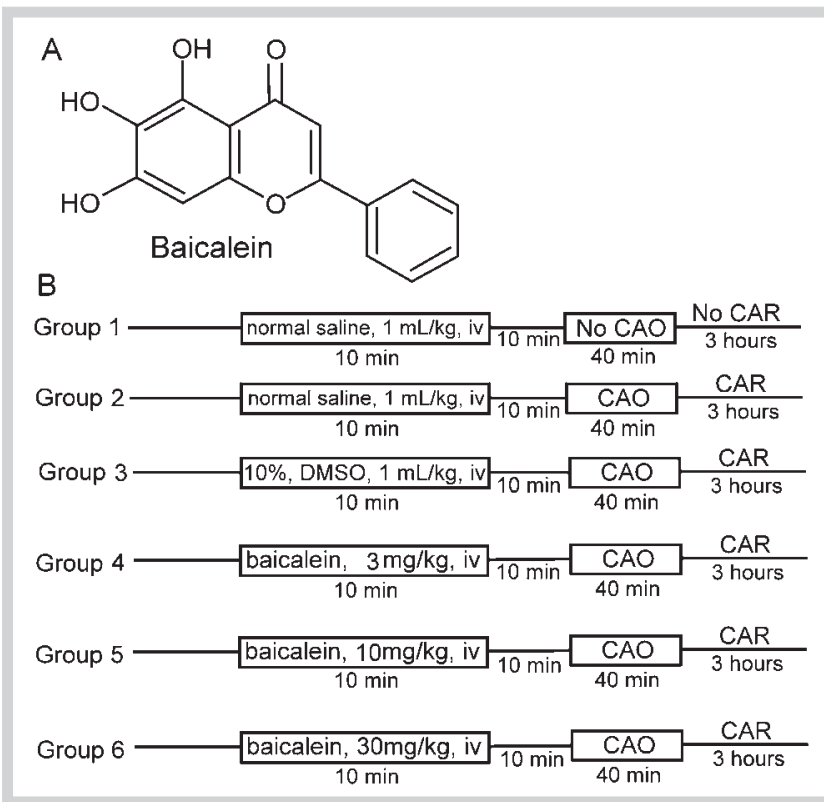

Fig. 1 A The chemical structure of baicalein. B The experimental protocol. Group 1 received the same surgical procedures and intravenous infusion of normal saline $(1 \mathrm{~mL} / \mathrm{kg})$ for 10 min without CAO or CAR. Group 2 received intravenous infusion of normal saline $(1 \mathrm{~mL} / \mathrm{kg})$ for $10 \mathrm{~min}$. Group 3 received intravenous infusion of $10 \%(\mathrm{v} / \mathrm{v})$ DMSO ( $1 \mathrm{~mL} / \mathrm{kg}$ ) for $10 \mathrm{~min}$. Group 4 received intravenous infusion of $3 \mathrm{mg} / \mathrm{kg}$ of baicalein for $10 \mathrm{~min}$. Group 5 received intravenous infusion of $10 \mathrm{mg} / \mathrm{kg}$ of baicalein for $10 \mathrm{~min}$. Group 6 received intravenous infusion of $30 \mathrm{mg} / \mathrm{kg}$ of baicalein for $10 \mathrm{~min}$. Ten minutes after the aforementioned pretreatments, all the rats except for those in Group 1 underwent a 40-min CAO and a 3-h CAR. Because there have not been any previous reports like our present study, there is no reference substance or treatment that has been previously established as having any effect when tested by the assay used in our present study. Therefore, no positive controls were included in the present study.

and liver diseases in Asia [7]. Lee et al. reported that baicalein improves cardiac contractile function in endotoxemic rats [8]. Baicalein has also been shown to exert anti-fibrotic effects in the rat liver [9]. However, the protective effects of baicalein on the kidneys after myocardial I/R are not known.

No specific therapy currently exists to prevent or treat AKI effectively [10]. A treatment for preventing AKI, however, might improve clinical outcomes. In this study, we investigated whether baicalein can reduce AKI induced by myocardial I/R. The roles of pro-survival kinases, including Akt and ERK1/2, as well as the roles of antiapoptotic Bcl-2 and proapoptotic Bax were also examined.

\section{Results}

$\nabla$

A total of 75 rats were randomly allocated into six groups (๑ Fig. 1B). The mortality rates were not significantly different $(p=0.718$, $\odot$ Table 1$)$. No significant changes were identified in arterial pressure and heart rate in any of the groups throughout the experiments. The hemodynamic variables did not differ significantly among the groups during the experiments ( $\bullet$ Table 2 ). Because there have not been any previous reports like our present study, i.e., reports investigating the protective effects of any substance or medicine against kidney injury induced by myocardial $\mathrm{I} / \mathrm{R}$, there is no reference substance or treatment that has previously been established as having any effect when tested by the assay used in our present study. Therefore, no positive controls were included in the present study.

The AAR and MI size of seven rats from each group except Group 1 were determined at the end of the experiments. The AAR values were not significantly different $(p=0.525$, $\odot$ Fig. $2 A)$. Baicalein at doses of 10 and $30 \mathrm{mg} / \mathrm{kg}$ significantly reduced the MI size ( $p<0.001$ vs. Group 2; $\odot$ Fig. 2 B). Baicalein at a dose of $3 \mathrm{mg} / \mathrm{kg}$ and DMSO (solvent vehicle) did not significantly change the MI size.

Blood was collected from seven rats in each group at the end of the experiments for biochemical analysis of kidney function. Myocardial I/R significantly increased the BUN level $(\mathrm{p}<0.001$ vs. Group 1). Baicalein and DMSO did not significantly reduce the increase of BUN ( $\odot$ Fig. 3A). Myocardial I/R also significantly increased the creatinine level ( $p<0.001$ vs. Group 1; 0 Fig. 3 B). However, pretreatment with baicalein at doses of 3,10 , and $30 \mathrm{mg} / \mathrm{kg}$ significantly reduced the increase of the creatinine level ( $p<0.001$ vs. Group 2). DMSO did not significantly limit the increase of the creatinine level.

At the end of the experiments, the kidneys of seven rats from each group were used for histological examinations of kidney injury. Myocardial I/R induced significant dilatation of Bowman's capsule ( $p<0.05$ vs. Group 1) and renal tubules $(p<0.01)$ ( Fig. 4). Baicalein at doses of 3,10 , and $30 \mathrm{mg} / \mathrm{kg}$ significantly limited the dilatation of Bowman's capsule ( $p<0.05$ vs. Group 2 ) and renal tubules $(\mathrm{p}<0.05)$. DMSO pretreatment did not significantly reduce the dilatation of Bowman's capsule and renal tubules.

At the end of the experiments, the kidneys of six rats from each group were used for TUNEL staining. TUNEL-positive nuclei were significantly increased in Group 2 ( $\mathrm{p}<0.001$ vs. Group 1; - Fig. 5). Baicalein at doses of 10 and $30 \mathrm{mg} / \mathrm{kg}$ significantly reduced the increase of TUNEL-positive nuclei ( $p<0.001$ vs. Group 2 ). Baicalein at a dose of $3 \mathrm{mg} / \mathrm{kg}$ also significantly limited the increase of TUNEL-positive nuclei ( $p<0.05$ ), although to a lesser extent than the larger doses. DMSO did not significantly change the increase of TUNEL-positive nuclei.

At the end of the experiments, the kidneys of another four rats from each group were used for Western blot analysis of activated caspase-3. Myocardial $\mathrm{I} / \mathrm{R}$ increased the activated caspase-3 (Lane 2, p < 0.001 vs. Group 1; ๑ Fig. 5C). Baicalein at doses of 10 and $30 \mathrm{mg} / \mathrm{kg}$ significantly inhibited the increase of activated caspase-3 (Lanes 5 and 6, p $<0.001$ vs. Group 2). Baicalein at a dose of $3 \mathrm{mg} / \mathrm{kg}$ also significantly reduced the increase of activated caspase-3 (Lane 4, p < 0.05). DMSO did not significantly limit the increase of activated caspase-3.

At the end of the experiments, blood was collected from five rats in each group to determine the serum levels of TNF- $\alpha$, IL- 1 , and IL-6. Compared to the sham group (i.e., Group 1), myocardial I/R significantly increased the serum levels of TNF- $\alpha$, IL-1, and IL-6 ( $\mathrm{p}<0.001$; Fig. 6). Baicalein at doses of 10 and $30 \mathrm{mg} / \mathrm{kg}$, however, significantly limited the increases in the serum levels of TNF- $\alpha$, IL-1, and IL-6 ( $p<0.001$ vs. Group 2). Baicalein at a dose of $3 \mathrm{mg} / \mathrm{kg}$ also significantly limited the increases in the serum levels of TNF- $\alpha(p<0.01)$, IL-1 $(p<0.001)$, and IL-6 $(p<0.001)$. DMSO did not significantly reduce the increases in the serum levels of TNF- $\alpha$, IL-1, and IL-6.

At the end of the experiments, the kidneys of four rats from each group were used for Western blot analysis of TNF- $\alpha$. Myocardial I/ $\mathrm{R}$ significantly increased TNF- $\alpha$ in Group 2 (Lane 2, $\mathrm{p}<0.01$ vs. Group 1; Fig. 6D). Baicalein at doses of 10 and $30 \mathrm{mg} / \mathrm{kg}$ signif- 
Table 1 Animal grouping and mortality. No significant differences in the mortality rates were observed among the groups. The Pearson's chi-squared p value was 0.718 . Rats in Group 1 received the same surgical procedures and intravenous infusion of normal saline $(1 \mathrm{~mL} / \mathrm{kg})$ for 10 min without coronary artery occlusion or reperfusion. Rats in Group 2 received intravenous infusion of normal saline $(1 \mathrm{~mL} / \mathrm{kg})$ for $10 \mathrm{~min}$. Rats in Group 3 received intravenous infusion of $10 \%(\mathrm{v} / \mathrm{v})$ DMSO $(1 \mathrm{~mL} / \mathrm{kg})$ for $10 \mathrm{~min}$. Rats in Group 4 received intravenous infusion of $3 \mathrm{mg} / \mathrm{kg}$ of baicalein for $10 \mathrm{~min}$. Rats in Group 5 received intravenous infusion of $10 \mathrm{mg} / \mathrm{kg}$ of baicalein for $10 \mathrm{~min}$. Rats in Group 6 received intravenous infusion of $30 \mathrm{mg} / \mathrm{kg}$ of baicalein for $10 \mathrm{~min}$. Ten minutes after the aforementioned pretreatments, all the rats except for those in Group 1 underwent a 40-min coronary artery occlusion and a 3-h reperfusion. Because there have not been any previous reports like our present study, there is no reference substance or treatment that has been previously established as having any effect when tested by the assay used in our present study. Therefore, no positive controls were included in the present study.

\begin{tabular}{|lllllll|} 
Group & $\begin{array}{l}\text { Treatment } \\
\text { Protocol }\end{array}$ & $\begin{array}{l}\text { Num- } \\
\text { ber }\end{array}$ & \multicolumn{2}{l}{ Mortality } & & $\begin{array}{l}\text { Number } \\
\text { Included }\end{array}$ \\
\hline 1 & Sham & 12 & 0 & 0 & 0 & 12 \\
\hline 2 & $\begin{array}{l}\text { Myocardial } \\
\text { I/R }\end{array}$ & 13 & 1 & 0 & 0 & 12 \\
\hline 3 & DMSO & 13 & 0 & 1 & 0 & 12 \\
\hline 4 & Bai*3 & 13 & 1 & 0 & 0 & 12 \\
\hline 5 & Bai*10 & 12 & 0 & 0 & 0 & 12 \\
\hline 6 & Bai*30 & 12 & 0 & 0 & 0 & 12 \\
\hline
\end{tabular}

Bai*3 = baicalein (3 mg/kg); Bai*10 = baicalein (10 mg/kg); Bai*30 = baicalein (30 mg/ $\mathrm{kg}) ; \mathrm{HF}=$ heart failure; $\mathrm{VF}=$ ventricular fibrillation icantly inhibited the increase of TNF- $\alpha$ (Lanes 5 and $6, \mathrm{p}<0.01$ and $\mathrm{p}<0.001$ vs. Group 2, respectively). Baicalein at a dose of $3 \mathrm{mg} / \mathrm{kg}$ also significantly limited the increase of TNF- $\alpha$ (Lane 4 , $\mathrm{p}<0.01$ ). DMSO did not significantly reduce the increase of TNF$\alpha$.

At the end of the experiments, the kidneys of four rats from each group were used for Western blot analysis of Bcl-2, Bax, phospho-Akt, and phospho-ERK1/2. Myocardial I/R significantly reduced Bcl-2 in Group 2 (Lane 2, $\mathrm{p}<0.05$ vs. Group 1; O Fig. 7 A). Baicalein at doses of 10 and $30 \mathrm{mg} / \mathrm{kg}$ significantly inhibited the decrease of Bcl-2 (Lanes 5 and $6, \mathrm{p}<0.05$ and $\mathrm{p}<0.001$ vs. Group 2 , respectively). Baicalein at a dose of $3 \mathrm{mg} / \mathrm{kg}$ did not significantly inhibit the decrease of Bcl-2.

Myocardial I/R significantly increased the density of the Bax bands in Group 2 (Lane 2, p $<0.01$ vs. Group 1; O Fig. 7 B). Baicalein at doses of 10 and $30 \mathrm{mg} / \mathrm{kg}$ significantly inhibited the increase of Bax (Lanes 5 and 6, p $<0.001$ vs. Group 2). Baicalein at a dose of $3 \mathrm{mg} / \mathrm{kg}$ also significantly reduced the increase of Bax (Lane 4, $\mathrm{p}<0.01$ ).

Myocardial I/R significantly reduced the phospho-Akt in Group 2 (Lane 2, p $<0.001$ vs. Group 1; O Fig. 7C). Baicalein at doses of 3 , 10 , and $30 \mathrm{mg} / \mathrm{kg}$ significantly prevented the decrease of phospho-Akt (Lanes 4, 5, and 6, p < 0.001 vs. Group 2).

Myocardial I/R significantly reduced phospho-ERK1/2 in Group 2 (Lane 2, p $<0.05$ vs. Group 1; $\odot$ Fig. 7D). Baicalein at doses of 10 and $30 \mathrm{mg} / \mathrm{kg}$ significantly inhibited the decrease of phosphoERK1/2 (Lanes 5 and $6, p<0.001$ vs. Group 2). Baicalein at a dose of $3 \mathrm{mg} / \mathrm{kg}$ also significantly prevented the decrease of phosphoERK1/2 (Lane 4, p < 0.05).

Table 2 Hemodynamic changes during the experiments. Values are mean \pm standard deviation. Rats in Group 1 received the same surgical procedure without any pretreatment, CAO, or CAR. The hemodynamic data shown for Group 1 were observed at the same time points as those for the other five groups. No significant changes were identified in mean arterial pressure and heart rate throughout the experiments. No significant differences were identified in the hemodynamic variables during the experiments.

\begin{tabular}{|c|c|c|c|c|c|c|c|c|c|}
\hline \multirow[t]{3}{*}{ Group } & \multirow{3}{*}{$\begin{array}{l}\text { Treatment } \\
\text { Protocol }\end{array}$} & \multirow[t]{3}{*}{ Number } & \multirow[t]{3}{*}{ Baseline 1} & \multirow[t]{3}{*}{ Baseline 2} & \multicolumn{2}{|l|}{ CAO } & \multicolumn{3}{|l|}{ CAR } \\
\hline & & & & & & & & & \\
\hline & & & & & $20 \mathrm{~min}$ & $40 \mathrm{~min}$ & $1 \mathrm{~h}$ & $2 \mathrm{~h}$ & $3 \mathrm{~h}$ \\
\hline \multicolumn{10}{|c|}{ Mean arterial pressure $(\mathrm{mmHg})$} \\
\hline 1 & Sham & 12 & $78 \pm 9$ & $80 \pm 7$ & $79 \pm 8$ & $74 \pm 5$ & $77 \pm 6$ & $76 \pm 4$ & $75 \pm 5$ \\
\hline 2 & Myocardial I/R & 12 & $76 \pm 6$ & $77 \pm 8$ & $75 \pm 5$ & $76 \pm 9$ & $76 \pm 7$ & $75 \pm 5$ & $74 \pm 6$ \\
\hline 3 & DMSO & 12 & $78 \pm 7$ & $80 \pm 7$ & $74 \pm 6$ & $77 \pm 7$ & $75 \pm 8$ & $75 \pm 5$ & $73 \pm 9$ \\
\hline 4 & Bai*3 & 12 & $78 \pm 6$ & $79 \pm 5$ & $73 \pm 5$ & $78 \pm 9$ & $77 \pm 6$ & $75 \pm 8$ & $77 \pm 9$ \\
\hline 5 & Bai*10 & 12 & $75 \pm 6$ & $80 \pm 6$ & $75 \pm 7$ & $78 \pm 5$ & $75 \pm 7$ & $75 \pm 6$ & $75 \pm 5$ \\
\hline 6 & Bai*30 & 12 & $80 \pm 9$ & $77 \pm 7$ & $76 \pm 5$ & $76 \pm 6$ & $77 \pm 10$ & $76 \pm 7$ & $73 \pm 8$ \\
\hline \multicolumn{10}{|c|}{ Heart rate (beats/min) } \\
\hline 1 & Sham & 12 & $423 \pm 44$ & $443 \pm 41$ & $431 \pm 36$ & $424 \pm 45$ & $439 \pm 44$ & $430 \pm 44$ & $418 \pm 52$ \\
\hline 2 & Myocardial I/R & 12 & $430 \pm 53$ & $425 \pm 39$ & $431 \pm 46$ & $426 \pm 50$ & $424 \pm 41$ & $428 \pm 41$ & $423 \pm 39$ \\
\hline 3 & DMSO & 12 & $429 \pm 52$ & $441 \pm 76$ & $421 \pm 61$ & $438 \pm 48$ & $422 \pm 45$ & $441 \pm 57$ & $428 \pm 59$ \\
\hline 4 & Bai*3 & 12 & $425 \pm 69$ & $440 \pm 60$ & $413 \pm 51$ & $439 \pm 49$ & $428 \pm 47$ & $423 \pm 49$ & $425 \pm 41$ \\
\hline 5 & Bai*10 & 12 & $429 \pm 41$ & $425 \pm 50$ & $423 \pm 65$ & $431 \pm 47$ & $427 \pm 50$ & $439 \pm 54$ & $438 \pm 62$ \\
\hline 6 & Bai*30 & 12 & $431 \pm 46$ & $427 \pm 40$ & $425 \pm 43$ & $435 \pm 41$ & $423 \pm 48$ & $427 \pm 40$ & $422 \pm 38$ \\
\hline \multicolumn{10}{|c|}{ Mean arterial pressure-heart rate product/1000 (mmHg*beats/min) } \\
\hline 1 & Sham & 12 & $33.14 \pm 5.56$ & $35.30 \pm 4.77$ & $33.96 \pm 4.50$ & $31.48 \pm 4.07$ & $33.66 \pm 4.22$ & $32.50 \pm 2.94$ & $31.45 \pm 3.71$ \\
\hline 2 & Myocardial I/R & 12 & $32.94 \pm 5.74$ & $32.66 \pm 5.06$ & $32.22 \pm 4.72$ & $32.64 \pm 6.55$ & $32.52 \pm 5.41$ & $31.99 \pm 4.29$ & $31.45 \pm 4.27$ \\
\hline 3 & DMSO & 12 & $33.35 \pm 5.85$ & $35.20 \pm 7.35$ & $31.17 \pm 5.49$ & $33.59 \pm 4.96$ & $31.34 \pm 4.12$ & $32.93 \pm 5.09$ & $31.19 \pm 5.93$ \\
\hline 4 & Bai*3 & 12 & $33.13 \pm 6.07$ & $34.77 \pm 4.89$ & $30.26 \pm 3.98$ & $34.17 \pm 6.47$ & $33.06 \pm 5.06$ & $31.77 \pm 5.23$ & $32.73 \pm 5.97$ \\
\hline 5 & Bai*10 & 12 & $32.19 \pm 4.33$ & $33.89 \pm 5.52$ & $32.21 \pm 7.40$ & $33.54 \pm 5.43$ & $32.23 \pm 5.92$ & $32.97 \pm 5.42$ & $32.68 \pm 5.58$ \\
\hline 6 & Bai*30 & 12 & $34.49 \pm 5.63$ & $32.87 \pm 5.32$ & $32.16 \pm 3.45$ & $33.20 \pm 4.39$ & $32.48 \pm 5.71$ & $32.38 \pm 4.83$ & $30.72 \pm 3.98$ \\
\hline
\end{tabular}

Bai*3 = baicalein $(3 \mathrm{mg} / \mathrm{kg})$; Bai* 10 = baicalein $(10 \mathrm{mg} / \mathrm{kg})$; Bai*30 = baicalein $(30 \mathrm{mg} / \mathrm{kg})$; Baseline 1 = baseline before treatment; Baseline 2 = baseline after treatment 

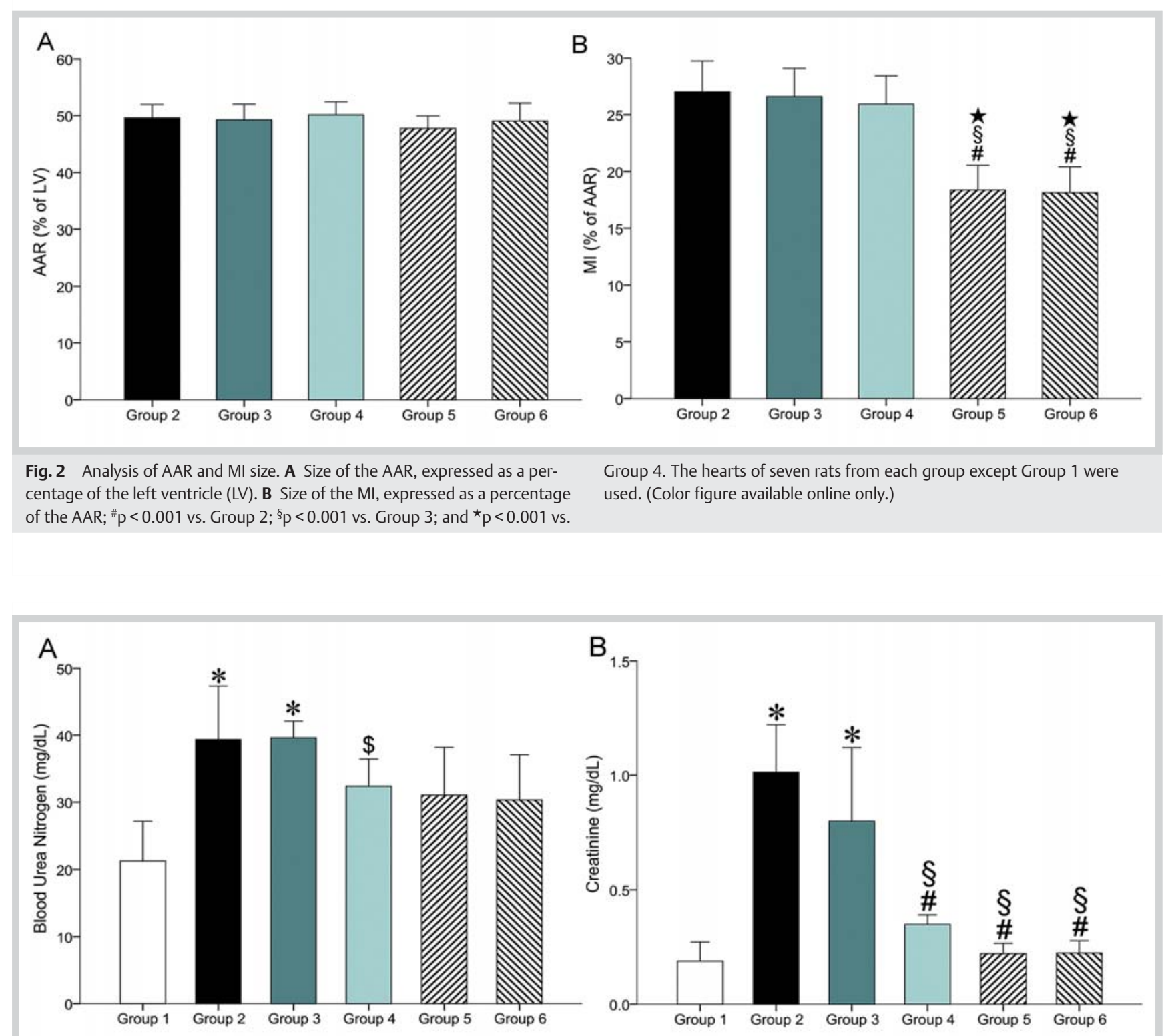

Fig. 3 Biochemical analysis of kidney function. A The blood urea nitrogen level. B The creatinine level; ${ }^{*} p<0.001$ vs. Group $1 ;{ }^{\$} p<0.05$ vs. Group 1 ;

${ }^{\#} \mathrm{p}<0.001$ vs. Group 2 ; and ${ }^{\S} \mathrm{p}<0.001$ vs. Group 3. Blood from seven rats from each group was collected. (Color figure available online only.)
DMSO did not significantly affect the changes of Bcl-2, Bax, phospho-Akt, and phospho-ERK1/2 in the kidneys induced by myocardial I/R.

\section{Discussion}

$\nabla$

In the current study, we demonstrated for the first time that baicalein attenuates kidney injury induced by myocardial I/R. Baicalein is a major component of the root of S. baicalensis, which has traditionally been used to treat cardiovascular and liver diseases in Asia [7]. The protective effects of baicalein on the heart and liver have been reported previously $[8,9]$. However, studies regarding the protective effects of baicalein on the kidneys are scant. Lee et al. reported that baicalein reduced the inflammatory responses induced by radiation in the mouse kidney [11]. Ahad et al. recently reported that baicalein prevented nephropathy in di- abetic rats [12]. This paper is the first report, however, on the protective effects of baicalein against AKI induced by myocardial I/R.

The mechanisms underlying the protective effects of baicalein on the kidneys are not fully understood. No significant hemodynamic changes were identified during the experiments, and hemodynamics did not differ significantly among the groups. As such, the inhibition of kidney injury and apoptosis by baicalein could not be ascribed to hemodynamic changes. Our study demonstrated that baicalein significantly reduced apoptosis in the kidneys. The serum levels of TNF- $\alpha$, IL-1, and IL- 6 , and the levels of TNF- $\alpha$ in the kidneys were significantly decreased. Baicalein significantly increased Bcl-2 and reduced Bax in the kidneys. Phosphorylation of Akt and ERK1/2 was significantly increased. Our findings suggested that the underlying mechanisms of the protection of baicalein against AKI might be related to the inhibition of apoptosis, 


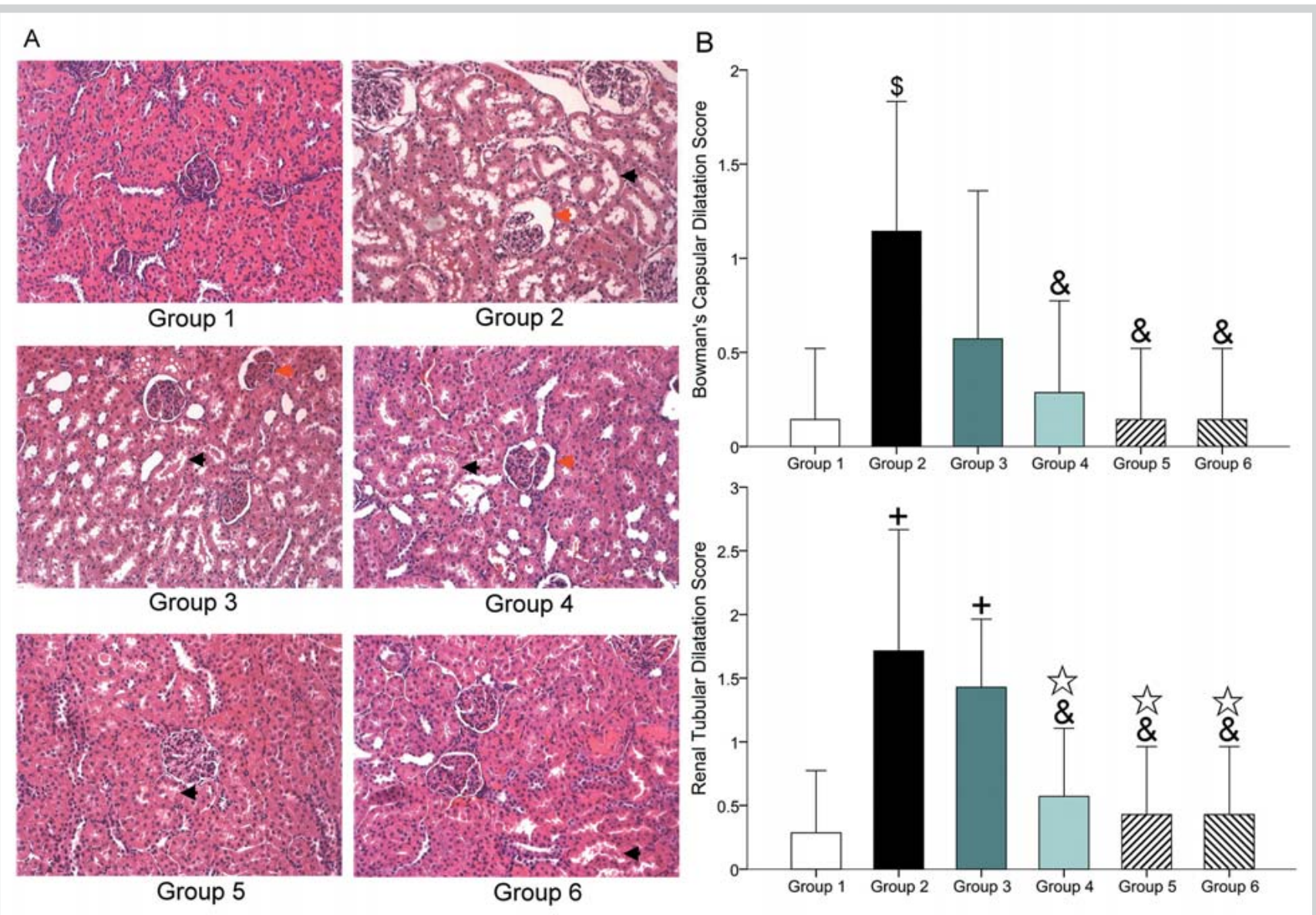

Fig. 4 Histological examination of kidney injury. A Representative photomicrographs of hematoxylin and eosin staining of kidney sections $(\times 200$ magnification). Significant dilatation of Bowman's capsule (red arrow) and renal tubules (black arrow) were found in the kidneys of the rats in Group 2.
B Histological scoring of dilatation of Bowman's capsule (upper panel) and renal tubules (lower panel); ${ }^{+} p<0.01$ vs. Group $1 ;{ }^{\$} p<0.05$ vs. Group 1 ; ${ }^{\&} p<0.05$ vs. Group 2 ; and ${ }^{\star} p<0.05$ vs. Group 3 . The kidneys of seven rats from each group were used. (Color figure available online only.) possibly through the inhibition of TNF- $\alpha$ production, the modulation of Bcl-2 and Bax, and the activation of Akt and ERK1/2.

Inhibition of apoptosis plays a crucial role in the protective effects of baicalein. Chen et al. recently demonstrated that baicalein prevented cardiac injury induced by lysophosphatidylcholine. The apoptosis was reduced in association with an increase of Bcl-2 and a decrease of Bax [13]. Huang et al. recently reported that baicalein dose-dependently reduced cell death in cardiomyocytes exposed to $\mathrm{H}_{2} \mathrm{O}_{2}$. DNA laddering was also attenuated [14]. AKI is a severe complication of acute MI and cardiac surgery, and a treatment for preventing AKI might significantly improve clinical outcomes. Venugopal et al. reported that remote ischemic preconditioning in the forearm reduces AKI in nondiabetic patients receiving coronary artery bypass grafting surgery [15]. Baicalin, another component of the root of S. baicalensis, has previously been reported to attenuate renal I/R injury [16].

In this study, we demonstrated that baicalein reduces kidney injury and apoptosis induced by myocardial I/R in a dose-dependent manner. Baicalein at doses of 10 and $30 \mathrm{mg} / \mathrm{kg}$ significantly reduced the MI size. Reduction of the MI size is associated with preserved heart function and decreased production of proinflammatory cytokines; both may contribute to the attenuation of kidney injury. However, baicalein at a dose of $3 \mathrm{mg} / \mathrm{kg}$ significantly limited kidney injury without significantly reducing the MI size. Taken together, these findings suggest that baicalein pro- tects against AKI induced by myocardial $I / R$, and that increased protective effects, which may be related to MI size reduction, are achieved at larger doses.

There are limitations in this study. No definite myocardial blood flow data were collected. However, rats have limited collateral coronary circulation, and our model is comparable to that widely used in experiments on myocardial I/R $[3,17]$. MI might affect arterial pressure and the blood flow to the kidneys. Baicalein reduces the MI size and thus might prevent the compromising of kidney blood flow. Although we did not specifically measure the arterial pressure or flow to the kidneys during the experiment, the arterial pressure measured via the femoral artery cannula did not differ significantly among the groups. Zhang et al. demonstrated that a lack of change in mean arterial pressure connotes a corresponding lack of change in renal blood flow [18].

In conclusion, we demonstrated that baicalein attenuates kidney injury induced by myocardial I/R. The underlying mechanisms might be related to the inhibition of apoptosis, possibly through the inhibition of TNF- $\alpha$ production, the modulation of the expression of Bcl-2 and Bax, and the activation of Akt and ERK1/2. Our findings elucidate the mechanisms of the protective effects of baicalein, and may provide a rationale for the application of baicalein to prevent AKI in acute MI and cardiac surgery. Studies on other animals and investigations into the roles of other signaling elements are necessary. 


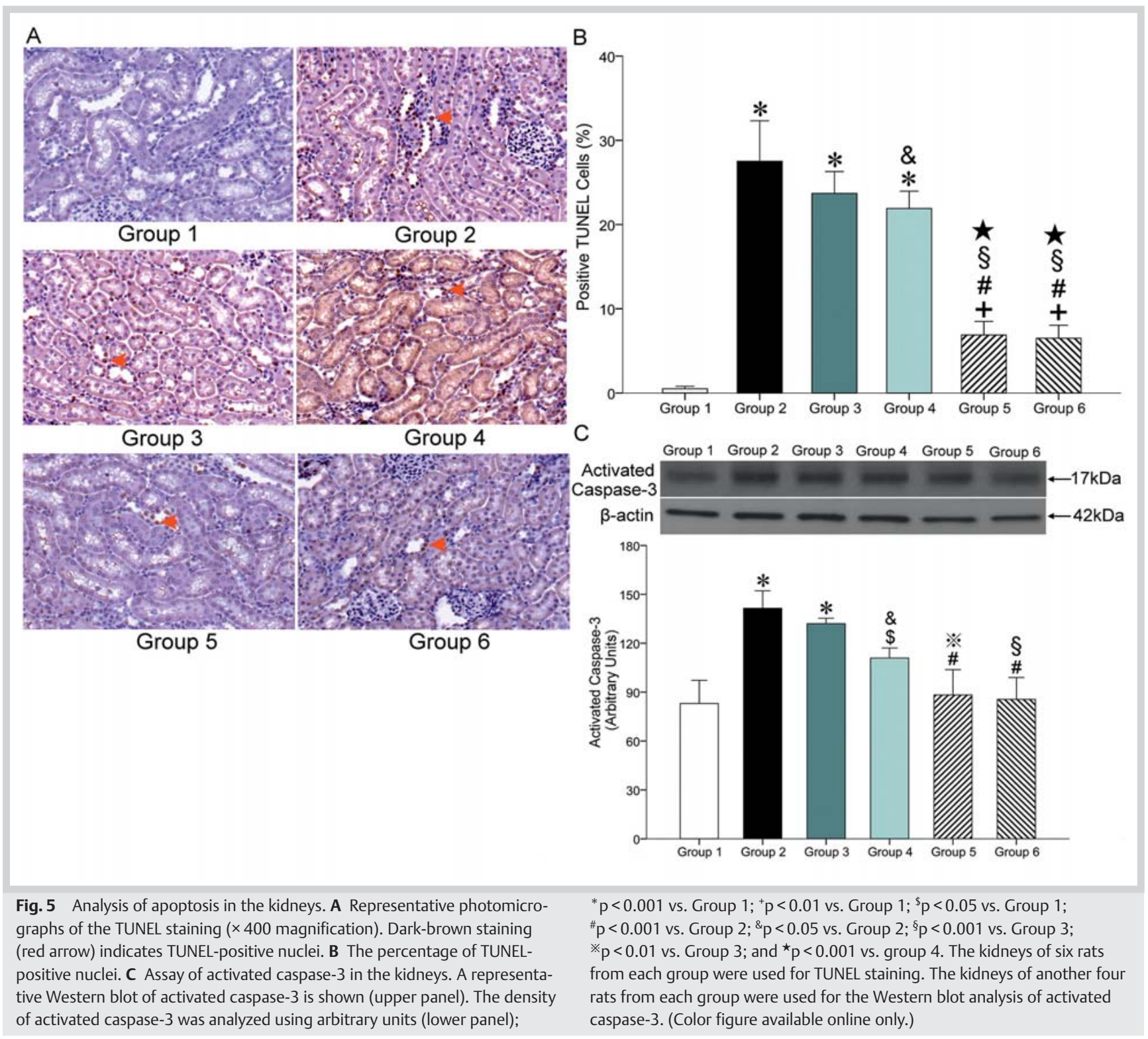

\section{Materials and Methods}

$\nabla$

Animals

Seventy-five male Sprague-Dawley rats (body weight: 250$280 \mathrm{~g}$ ) were purchased from the National Laboratory Animal Breeding and Research Center of the Ministry of Science and Technology. All animals were housed at an ambient temperature of $23 \pm 1{ }^{\circ} \mathrm{C}$ and a humidity of $55 \pm 5 \%$. All animals were cared for humanely in accordance with the Guide for the Care and Use of Laboratory Animals (National Academic Press, USA, 1996). This study was approved by the Animal Experiment Committee of Taipei Veterans General Hospital (approval number IACUC 2014078; approval date June 16, 2014).

\section{Chemical and reagents}

Baicalein (purity 98\%) was purchased from Sigma-Aldrich. Baicalein was dissolved in DMSO (Sigma-Aldrich) and diluted to the desired concentrations in normal saline. The final concentration of DMSO in the injected solution was $10 \%(\mathrm{v} / \mathrm{v})$. The diamino- benzidine-chromogen used for TUNEL staining was purchased from Boehringer.

\section{Animal preparation}

The techniques of animal preparation used in this study have been previously reported [3]. Briefly, each rat was anesthetized. The femoral artery was cannulated. Electrocardiography leads were placed. After median sternotomy, a 4-0 silk suture was passed around the proximal left anterior descending coronary artery. The ends of the silk suture were threaded through a small vinyl tube to form a snare. Body temperature was maintained at $37^{\circ} \mathrm{C}$ throughout the experiments.

\section{Experimental protocol}

A total of 75 rats were randomly allocated into six groups ( Fig. 1B). Group 1 rats received the surgical procedure described above in addition to intravenous infusion of normal saline ( $1 \mathrm{~mL} / \mathrm{kg}$ ) for $10 \mathrm{~min}$ without coronary artery occlusion or reperfusion that would be administered to the other groups (sham group, 12 rats). Group 2 rats received intravenous infusion of 


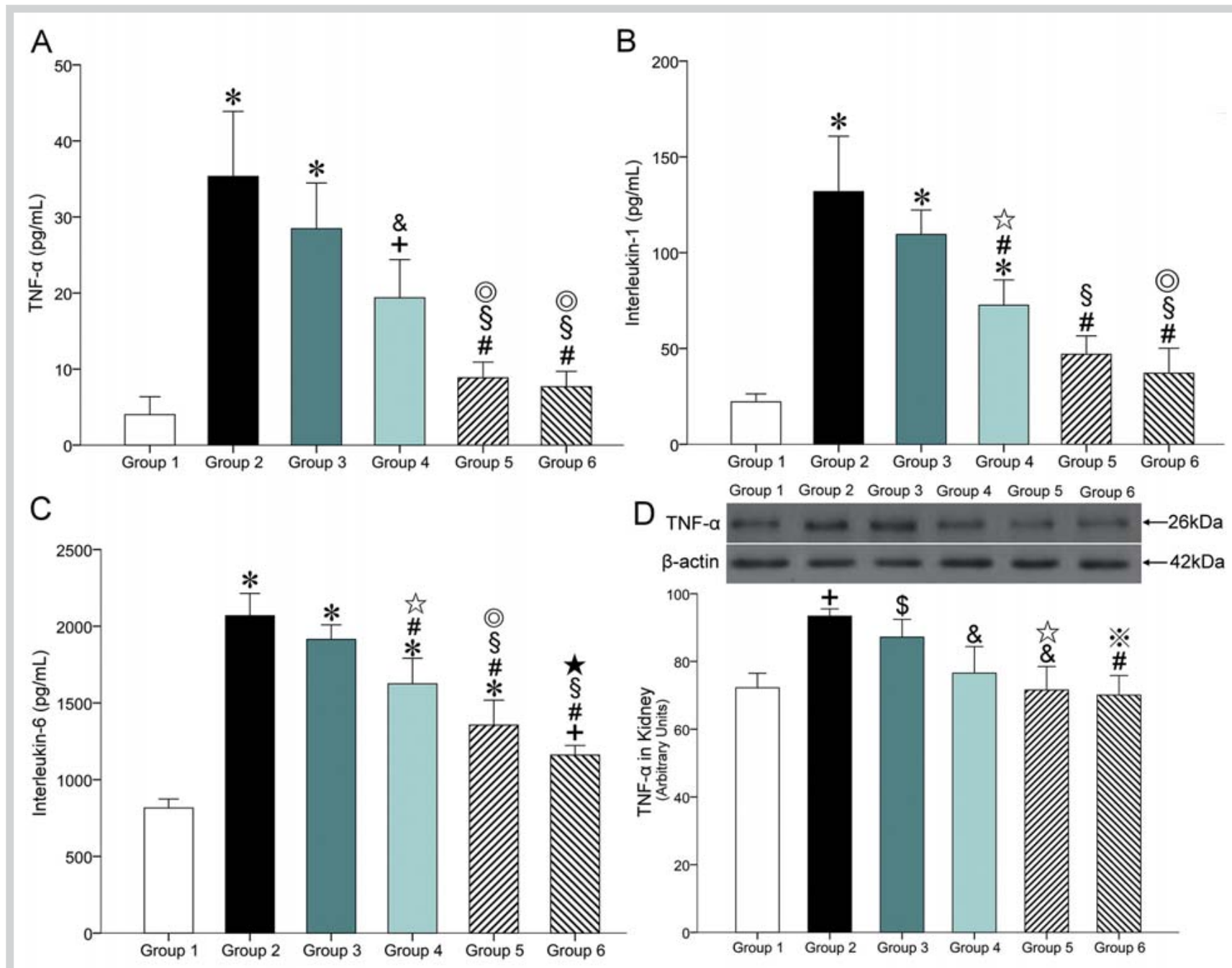

Fig. 6 A Serum level of TNF- $\alpha$. B Serum level of IL-1. C Serum level of IL-6. D Assay of TNF- $\alpha$ in the kidneys. Representative Western blots of TNF- $\alpha$ are shown (upper panel). The density of TNF- $\alpha$ was analyzed using arbitrary units (lower panel); ${ }^{*} p<0.001$ vs. Group $1 ;{ }^{+} p<0.01$ vs. Group $1 ;{ }^{\$} p<0.05$ vs. Group 1; ${ }^{\#} p<0.001$ vs. Group 2; ${ }^{\&} p<0.01$ vs. Group $2 ;{ }^{\S} p<0.001$ vs. Group 3;

${ }^{*} p<0.01$ vs. Group 3; $\mathrm{k} p<0.05$ vs. Group 3 ; ${ }^{\star} \mathrm{p}<0.001$ vs. Group 4; and ${ }^{\circ} p<0.05$ vs. Group 4 . Blood was collected from five rats from each group to determine the serum levels of TNF- $\alpha$, IL-1, and IL-6. The kidneys of four rats from each group were used for the Western blot analysis of TNF- $\alpha$. (Color figure available online only.) normal saline $(1 \mathrm{~mL} / \mathrm{kg}$ ) for $10 \mathrm{~min}$ (myocardial I/R group, 13 rats). Group 3 rats received intravenous infusion of $10 \%(\mathrm{v} / \mathrm{v})$ DMSO ( $1 \mathrm{~mL} / \mathrm{kg}$ ) for $10 \mathrm{~min}$ (DMSO group, 13 rats). Group 4 rats received intravenous infusion of $3 \mathrm{mg} / \mathrm{kg}$ of baicalein for $10 \mathrm{~min}$ (baicalein*3 group, 13 rats). Group 5 rats received intravenous infusion of $10 \mathrm{mg} / \mathrm{kg}$ of baicalein for $10 \mathrm{~min}$ (baicalein*10 group, 12 rats). Group 6 rats received intravenous infusion of $30 \mathrm{mg} / \mathrm{kg}$ of baicalein for $10 \mathrm{~min}$ (baicalein*30 group, 12 rats). Ten minutes after the aforementioned pretreatments, the rats in all of the groups except Group 1 received a 40-min coronary artery occlusion by pulling the snare around the proximal left anterior descending coronary artery. Successful occlusion was verified by observing the development of ST-segment elevation and changes in the QRS complex on electrocardiograms and the cyanotic change of the occluded myocardium. Subsequently, the snare was released for reperfusion for $3 \mathrm{~h}$. Reperfusion was confirmed by refilling the coronary artery and observing a reactive hyperemic response. Arterial pressure, heart rate, and electrocardiograms were recorded simultaneously and continuously throughout the experiments.

\section{Determination of the area at risk and myocardial infarct size}

At the end of the experiments, 1000 units of heparin were intravenously administered. The heart was excised. The coronary artery was ligated at the site of previous occlusion. The ascending aorta was perfused with $1 \%$ Evans blue dye. The left ventricle plus septum was cut into 6-7 transverse slices, which were incubated at $37^{\circ} \mathrm{C}$ in $1 \%$ triphenyl tetrazolium chloride solution for $20 \mathrm{~min}$. The slices were weighed and fixed in $10 \%$ formalin solution for $24 \mathrm{~h}$. The basal surfaces were photographed. The AAR and MI of each heart slice were calculated by planimetry. The total weights of the AAR and MI were calculated and summed. The AAR was reported as a percentage of the left ventricle plus septum, with the MI size as a percentage of the AAR [19].

\section{Biochemical analysis of kidney function}

BUN and creatinine levels were measured using colorimetric methods adapted to an autoanalyzer and expressed as $\mathrm{mg} / \mathrm{dL}$. 

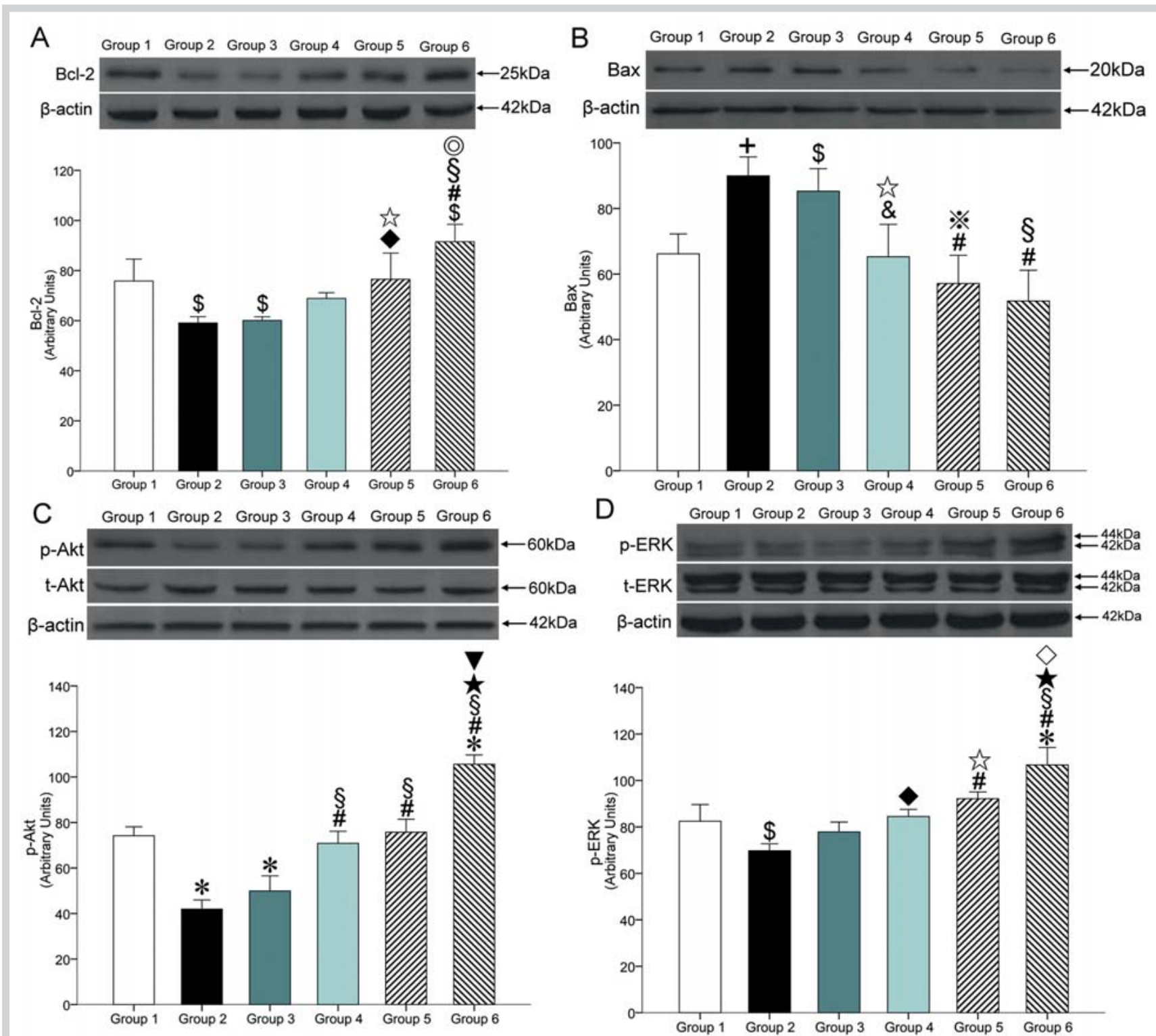

Fig. 7 A Bcl-2 assay. B Bax assay. C Phospho-Akt (p-Akt) and total Akt (tAkt) assay. D Phospho-extracellular signal-regulated kinases ( $p$-ERK) and total ERK (t-ERK) assay. Representative Western blots of Bcl-2, Bax, p-Akt, t-Akt, $\mathrm{p}$-ERK, and t-ERK are shown (upper panel). The densities of Bcl-2, Bax, p-Akt, and $\mathrm{p}$-ERK bands were analyzed using arbitrary units (lower panel);

${ }^{*} p<0.001$ vs. Group $1 ;{ }^{+} p<0.01$ vs. Group $1 ;{ }^{\$} p<0.05$ vs. Group $1 ;$

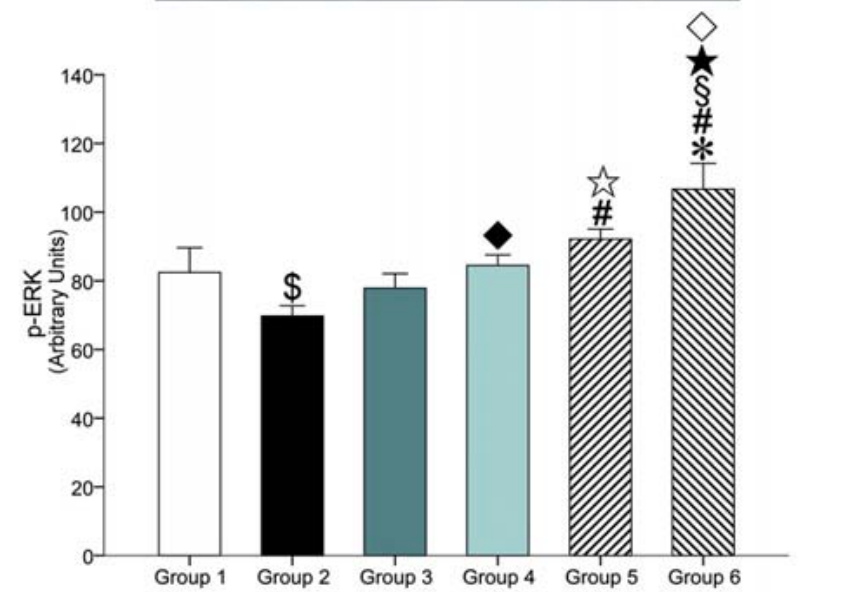

${ }^{\#} \mathrm{p}<0.001$ vs. Group $2 ;{ }^{\&} \mathrm{p}<0.01$ vs. Group $2 ;{ }^{\dagger} \mathrm{p}<0.05$ vs. Group 2; ${ }^{\S} p<0.001$ vs. Group 3; ${ }^{*} p<0.01$ vs. Group 3; ${ }^{2} p<0.05$ vs. Group 3; ${ }^{\star} \mathrm{p}<0.001$ vs. Group 4; ${ }^{\odot} \mathrm{p}<0.01$ vs. Group 4; $\mathrm{p}<0.001$ vs. Group 5; and $\diamond p<0.05$ vs. Group 5 . The kidneys of four rats from each group were used for the Western blot analysis of Bcl-2, Bax, p-Akt, t-Akt, p-ERK, and t-ERK. (Color figure available online only.)

\section{Histological examination of kidney injury}

The kidneys were fixed in $10 \%$ buffered formaldehyde, embedded, and sectioned in $5 \mu \mathrm{m}$ slices. These sections were stained with hematoxylin and eosin. A histological evaluation was performed in a blinded manner by a pathologist on the dilatation of Bowman's capsule and renal tubules. Histological changes were scored on a 4-point scale. The score was 0 if there were no changes. The score was 1 if the changes were mild and focal. The score was 2 if the changes were intermediate and multifocal. The score was 3 if the changes were prominent and extensive [20].

\section{TUNEL staining of the kidneys}

The methods of TUNEL staining used in this study have been previously reported [3]. Briefly, the kidneys were sectioned and fixed in acetone. Samples were incubated with terminal deoxynucleotidyl transferase and detection buffer conjugated with horseradish peroxidase. A diamino-benzidine-chromogen was used. The number of TUNEL-positive nuclei was counted in randomly selected areas and expressed as a percentage of the total number of cellular nuclei at a magnification of $\times 400$. 
Determination of the serum levels of tumor necrosis factor- $\alpha$, interleukin-1, and interleukin- 6

The serum levels of TNF- $\alpha$, IL-1, and IL- 6 were determined through ELISA by using a commercially available ELISA set. ELISA was performed according to the manufacturer's instructions. All samples and standards were measured in duplicate.

\section{Western blot analysis for caspase-3 activation, TNF- $\alpha$,}

\section{$\mathrm{BCl}-2$, Bax, Akt, and ERK1/2 in the kidneys}

The methods of Western blot analysis used in this study have been previously reported [3]. Briefly, the kidneys were homogenized at $4^{\circ} \mathrm{C}$. Equivalent amounts of protein samples were separated using electrophoresis and transferred to a nitrocellulose membrane. The membranes were incubated with various appropriate antibodies at $1: 1000(\mathrm{v} / \mathrm{v})$ dilution in $5 \%$ nonfat dry milk. The membranes were incubated with secondary antibody conjugated to horseradish peroxidase. Peroxidase activity was visualized, followed by exposure to hyperfilms. $\beta$-actin was detected as a loading control for protein quantity. The optical density for each band was determined using NIH Image 1.6 and was normalized against the background density for each gel.

\section{Statistical analysis}

All values are expressed as mean \pm standard deviation. Hemodynamic variables were analyzed using two-way analysis of variance with repeated measures. Multiple comparisons of repeated measures were performed using within-subject contrasts. The data were analyzed using one-way analysis of variance and the Bonferroni post hoc multiple comparison test. The ordinal values of the kidney injury scores were analyzed using the Mann-Whitney nonparametric test. Differences were considered significant at $\mathrm{p}$ values of less than 0.05 .

\section{Acknowledgments}

This study was supported by grants from the National Science Council (NSC 102-2314-B-075-020) and Taipei Veterans General Hospital (V102 C-038), Taiwan. This work was assisted in part by the Division of Experimental Surgery, Department of Surgery, Taipei Veterans General Hospital. The authors thank Shiang-Rong Jeang for her excellent technical support in the experiments.

\section{Conflict of Interest}

The authors declare no conflicts of interest.

\section{Affiliations}

${ }^{1}$ Division of Cardiovascular Surgery, Department of Surgery, Taipei Veterans General Hospital, Taipei, Taiwan

2 Division of Cardiovascular Surgery, Department of Surgery, National YangMing University School of Medicine, Taipei, Taiwan

${ }^{3}$ Institute of Clinical Medicine, National Yang-Ming University School of Medicine, Taipei, Taiwan

${ }^{4}$ Division of Cardiology, Department of Medicine, Taipei Veterans General Hospital, Taipei, Taiwan

${ }^{5}$ Cardiovascular Research Center, National Yang-Ming University, Taipei, Taiwan

${ }^{6}$ Department of Pathology and Laboratory Medicine, Taipei Veterans General Hospital, Taipei, Taiwan

${ }^{7}$ Department of Pathology and Laboratory Medicine, National Yang-Ming University School of Medicine, Taipei, Taiwan

${ }^{8}$ Institute of Hospital and Health Care Administration, National Yang-Ming University School of Medicine, Taipei, Taiwan
${ }^{9}$ Department of Physical Education and Health, University of Taipei, Taipei, Taiwan

\section{References}

1 Fox CS, Muntner P, Chen AY, Alexander KP, Roe MT, Wiviot SD. Short-term outcomes of acute myocardial infarction in patients with acute kidney injury: a report from the national cardiovascular data registry. Circulation 2012; 125: 497-504

2 Dardashti A, Ederoth P, Algotsson L, Brondén B, Bjursten H. Incidence, dynamics, and prognostic value of acute kidney injury for death after cardiac surgery. J Thorac Cardiovasc Surg 2014; 147: 800-807

3 Huang CH, Lai CC, Yang AH, Chiang SC. Myocardial preconditioning reduces kidney injury and apoptosis induced by myocardial ischaemia and reperfusion. Eur J Cardiothorac Surg 2015; 48: 382-391

4 Frangogiannis NG, Smith CW, Entman ML. The inflammatory response in myocardial infarction. Cardiovasc Res 2002; 53: 31-47

5 Baud V, Karin M. Signal transduction by tumor necrosis factor and its relatives. Trends Cell Biol 2001; 11: 372-377

6 Lee HT, Kim JY, Kim M, Wang P, Tang L, Baroni S, D’Agati VD, Desir GV. Renalase protects against ischemic AKI. J Am Soc Nephrol 2013; 24: 445-455

7 Huang Y, Tsang SY, Yao X, Chen ZY. Biological properties of baicalein in cardiovascular system. Curr Drug Targets Cardiovasc Haematol Disord 2005; 5: 177-184

8 Lee YM, Cheng PY, Chim LS, Kung CW, Ka SM, Chung MT, Sheu JR. Baicalein, an active component of Scutellaria baicalensis Georgi, improves cardiac contractile function in endotoxaemic rats via induction of heme oxygenase- 1 and suppression of inflammatory responses. J Ethnopharmacol 2011; 135: 179-185

9 Sun $H$, Che QM, Zhao X, Pu XP. Antifibrotic effects of chronic baicalein administration in a CCl4 liver fibrosis model in rats. Eur J Pharmacol 2010; 631: 53-60

10 Jo SK, Rosner MH, Okusa MD. Pharmacologic treatment of acute kidney injury: why drugs haven't worked and what is on the horizon. Clin J Am Soc Nephrol 2007; 2: 356-365

11 Lee EK, Kim JM, Choi J, Jung KJ, Kim DH, Chung SW, Ha YM, Yu BP, Chung $H Y$. Modulation of NF- $\kappa$ B and FOXOs by baicalein attenuates the radiation-induced inflammatory process in mouse kidney. Free Radic Res 2011; 45: 507-517

12 Ahad A, Mujeeb M, Ahsan H, Siddiqui WA. Prophylactic effect of baicalein against renal dysfunction in type 2 diabetic rats. Biochimie 2014; 106: $101-110$

13 Chen HM, Hsu JH, Liou SF, Chen TJ, Chen LY, Chiu CC, Yeh JL. Baicalein, an active component of Scutellaria baicalensis Georgi, prevents lysophosphatidylcholine-induced cardiac injury by reducing reactive oxygen species production, calcium overload and apoptosis via MAPK pathways. BMC Complement Altern Med 2014; 14: 233

14 Huang HH, Shao ZH, Li CQ Vanden Hoek TL, Li J. Baicalein protects cardiomyocytes against mitochondrial oxidant injury associated with JNK inhibition and mitochondrial Akt activation. Am J Chin Med 2014; 42: 79-94

15 Venugopal V, Laing CM, Ludman A, Yellon DM, Hausenloy D. Effect of remote ischemic preconditioning on acute kidney injury in nondiabetic patients undergoing coronary artery bypass graft surgery: a secondary analysis of 2 small randomized trials. Am J Kidney Dis 2010; 56: 10431049

16 Lin M, Li L, Li L, Pokhrel G, Qi G, Rong R, Zhu T. The protective effect of baicalin against renal ischemia-reperfusion injury through inhibition of inflammation and apoptosis. BMC Complement Altern Med 2014; 14: 19

17 Huang HH, Lai CC, Chiang SC, Chang SC, Chang CH, Lin JC, Huang CH. Brief left ventricular pressure overload reduces myocardial apoptosis. J Surg Res 2015; 194: 34-42

18 Zhang L, Shao X, Zhou C, Guo X, Jin L, Lian L, Yu X, Dong Z, Mo Y, Fang J. Transcutaneous electrical nerve stimulation regulates organ blood flow and apoptosis during controlled hypotension in dogs. PLoS One 2014; 9: e94368

19 Huang CH, Tsai SK, Chiang SC, Wang YY, Chih CL, Weng ZC, Lai ST. Brief pressure overload preconditions rabbit myocardium independent of adenosine receptor activation. Ann Thorac Surg 2011; 92: 1727-1732

20 Gulmen S, Kiris I, Narin C, Ceylan BG, Mermi B, Sutcu R, Meteoglu I. Tezosentan reduces the renal injury induced by abdominal aortic ischemiareperfusion in rats. J Surg Res 2009; 157: e7-e13 